\title{
UTILIZAÇÃO DA CIANOBACTÉRIA Spirulina maxima E DA LEVEDURA Saccharomyces cerevisiae COMO DIETAS COMPLEMENTARES NO CULTIVO DE Artemia franciscana
}

\author{
I. S. SOUZA ${ }^{1 *}$ e P. H. C. OLIVEIRA ${ }^{2}$ \\ ${ }^{1}$ Instituto Federal de Educação Ciência e Tecnologia do Rio Grande do Norte - IFRN \\ ${ }^{2}$ Instituto Federal de Educação Ciência e Tecnologia do Acre - IFAC \\ ivanildo.surini@ifrn.edu.br*
}

Artigo submetido em maio/2012 e aceito em maio/2015

DOI: $10.15628 /$ holos. 2015.925

\section{RESUMO}

Diferentes combinações da cianobactéria S. maxima e da levedura $S$. cerevisiae foram utilizadas no cultivo de $A$. franciscana, com o objetivo de avaliar dietas de baixo custo e fácil manuseio, e que mantivessem as larvas desse microcrustáceo em laboratório, de modo a propiciar estudos de sua ecologia, biologia e uso na aquicultura. Para isso, 12 cones de Imhoff contendo $1 \mathrm{~L}$ de água do mar filtrada foram estocados com 100 náuplios e submetidos à aeração contínua, monitoramento do $\mathrm{pH}$, temperatura, salinidade, oxigênio dissolvido e luminosidade, além de renovação diária de $50 \%$ do volume de água. Durante dez dias, os náuplios foram alimentados duas vezes ao dia com $25 \mathrm{mg}$ de diferentes combinações de $S$. maxima e $S$. cerevisiae, nas seguintes proporções: 100/0; 75/25; 50/50; 25/75 e $0 / 100$. Foram avaliadas a taxa de sobrevivência, proporção sexual, proporção juvenis/adultos e performance reprodutiva. Os resultados indicaram um melhor desempenho zootécnico de $A$. franciscana nos tratamentos que associaram S. maxima à $S$. cerevisiae (75/25, 50/50 e 25/75), e desta maneira, demonstram que a referida mistura dietética, em tais proporções, é viável para manter as larvas em cultivo laboratorial.

PALAVRAS-CHAVE: Artemia, cultivo, levedura, cianobactéria.

\section{USE THE CYANOBACTERIUM Spirulina maxima AND YEAST Saccharomyces cerevisiae AS FURTHER IN DIETS FOR CULTIVATION OF Artemia franciscana}

\begin{abstract}
Different combinations of cyanobacterium $S$. maxima and yeast $S$. cerevisiae were used in the cultivation of $A$. franciscana, with the objective of evaluate low cost of the diets, the easy handling, and also the Artemia larvae maintenance in laboratory, to propitiate studies about Artemia ecology, biology and its use in the aquaculture. For that, 12 Imhoff cones filled with $1 \mathrm{~L}$ of filtered sea water were stocked with 100 nauplii and submitted to the continuous aeration, $\mathrm{pH}$ monitorring, temperature, salinity, luminosity and dissolved oxygen values, besides the daily change of $50 \%$ of the water volume. For ten
\end{abstract}

days, nauplii were fed twice a day, $25 \mathrm{mg}$ in each time of different proportions of $S$. maxima and $S$. cerevisiae, in the following proportions: 100/0; 75/25; 50/50; 25/75 and $0 / 100$. The survival rate, sex ratio, juvenals/adults ratio and reproductive performance were evaluated. The results indicated a better performance in the treatments which associated $S$. maxima to $S$. cerevisiae $(75 / 25,50 / 50$ and $25 / 75$ ), and therefore they demonstrated that the referred dietary mixture, in such proportions, is viable to maintain $A$. franciscana larvae in laboratory culture.

KEYWORDS: Artemia, cultivation, yeast, cyanobacterium. 


\section{INTRODUÇÃO}

Nos últimos anos, o microcrustáceo Artemia vem desempenhando um papel central no desenvolvimento da aquicultura. A maior razão para o seu interesse é o seu relevante papel como alimento vivo e de uso prático em aquicultura, dado seu valor e plasticidade nutricional, facilidade no manejo, características de desenvolvimento, pequeno tamanho dos naúplios e metanaúplios, e longa vida de prateleira desempenhada pelos seus ovos císticos (Szyper, 2003).

Além disso, ao contrário de outros zooplactontes potencialmente úteis, as formas adultas de Artemia têm o exoesqueleto extremamente delgado e de fácil digestão para os predadores. Assim, tanto em sua forma larval (naúplios eclodidos a partir de cistos) como no estágio adulto (biomassa), esse microcrustáceo é considerado o mais versátil e popular de todos os alimentos usados em aquicultura. Ele é utilizado na alimentação de larvas e juvenis de peixes e camarões, da mesma forma que na indução à reprodução e na engorda de indivíduos adultos. Ainda, Artemia tem sido usado como vetor para bioencapsular - via emulsões lipídicas - vários componentes profiláticos e nutricionais em um número crescente de organismos aquáticos (Persoone e Sorgeloos, 1980).

Os cistos de Artemia, que na realidade são embriões inativos, se encontram comercialmente disponíveis nos mercados especializados em aquicultura. Em particular, o Great Salt Lake (Utah, EUA) dispõe de mais de 90 \% do mercado internacional de cistos, sendo que este produto é resultado da atividade de extração das populações naturais existentes naquele corpo d'água. Pelo fato de o equilíbrio destas populações está sujeito a variações ambientais, sobretudo de origem climática, a oferta de cistos pode flutuar bastante em determinados anos (Vinatea, 1998).

Sem dúvida, um aumento no setor aquícola somado às intempéries ambientais que diminuem as populações de Artemia, provocam efeitos muito prejudiciais para o bom desempenho da aquicultura. Tais fatos tornam a oferta de cistos e de seus produtos alternativos (biomassa viva, congelada, liofilizada, "flakes", "pellets") incapazes de suprir a grande demanda existente na atualidade.

A importância do valor nutritivo de Artemia para alimentação de organismos marinhos, reside em sua composição de ácidos graxos altamente insaturados da série omega 3 (HUFAs w3). O perfil de ácidos graxos HUFAs w3 deste organismo é altamente variável, dependendo da cepa geográfica e da dieta a qual está submetida determinada população. Apesar disso, este perfil é insuficiente para manter um ótimo crescimento e sobrevivência das larvas de organismos marinhos. Por esse motivo, atualmente vem se desenvolvendo várias técnicas para o enriquecimento do valor nutritivo de Artemia por meio da bioencapsulação de componentes essenciais (Tizol, 1994).

Vários experimentos demonstram que esse microcrustáceo pode ser cultivado com diferentes tipos de alimento inerte; já que a seleção de seus alimentos baseia-se unicamente no tamanho da partícula - até $50 \mu \mathrm{m}$. Tais alimentos podem substituir as microalgas, sua principal fonte alimentar, e desta maneira fazer com que a produção de adultos seja economicamente mais viável. Esses, além de diminuir significativamente os custos, simplifica marcadamente os processos 
de obtenção de biomassa. Entre esses produtos encontra-se farinha de trigo, pó de arroz e de soja, macroalgas, levedura de cerveja e de padaria e melaço de cana.

Contudo as algas unicelulares, em escala laboratorial, ainda permanecem como um alimento indispensável para a criação de organismos aquáticos filtradores. Porém, o cultivo de tais microalgas é de alto custo e necessita suporte intensivo. Assim sendo, o desenvolvimento para substitutos de microalgas vivas é de importância primária nos sistemas de cultivo.

Nesse sentido, a cianobactéria S. maxima e a levedura $S$. cerevisiae oferecem várias características interessantes, tais como: aquisição a partir de materiais com baixo custo de produção, partículas de tamanho satisfatório, o que os torna mais facilmente assimiláveis pela Artemia, e um elevado teor proteico. Além disso, a rígida parede celular presente em S. cerevisiae previne a lixiviação de nutrientes no meio de cultura e subsequentemente a deterioração da qualidade da água. A levedura, em suspensão na água, ainda pode servir para agregar outras fontes alimentares (bactérias e microalgas). Já S. maxima, propicia uma boa flutuabilidade na coluna d'água, alta digestibilidade, graças a presença de uma cadeia simples de aminoácidos, e uma fina parede celular formada por mucopolissacarídeos não tóxicos (Coutteau et. al., 1992; Pedraza, 1989).

Por fim, a produção da cianobactéria S. maxima e da levedura S. cerevisiae também tem sido incluída substancialmente como uma fonte proteica na mistura de dietas para a produção da biomassa de Artemia e larvas de outros crustáceos (Coutteau et. al., 1992).

A necessidade de dispor suficiente quantidades de Artemia, para atender a alimentação de animais cultivados em laboratórios e/ou em tanques ao ar livre, de desenvolver uma dieta de baixo custo e fácil manuseio, que mantenha as larvas em laboratório para estudo de sua ecologia e biologia, estimula o desenvolvimento de projetos que visam substitutos para as microalgas vivas na dieta desse microcrustáceo portador de considerável importância biológica e comercial.

Diante dessa necessidade, o presente trabalho tem como objetivo avaliar a utilização da cianobactéria S. maxima e da levedura S. cerevisiae como dietas complementares no cultivo de $A$. franciscana em escala laboratorial.

\section{MATERIAIS E MÉTODOS}

Cistos de A. franciscana provenientes de Grossos/RN foram levados ao Laboratório de Nutrição Aquática (LNAqua), Departamento de Oceanografia e Limnologia da Universidade Federal do Rio Grande do Norte (UFRN). Em laboratório, $1 \mathrm{~g}$ de cistos foi devidamente pesado e, em seguida, transferido para um cone de sedimentação de Imhoff preenchido com $1 \mathrm{~L}$ de água do mar previamente filtrada em rede de zooplâncton com malha de $120 \mu \mathrm{m}$. As condições de incubação para a eclosão dos naúplios se enquadraram naquelas preconizadas por Sorgeloos et al. (1986): salinidade de $35 \%$, oxigênio dissolvido acima de $5 \mathrm{mg} / \mathrm{L}$, luminosidade superior a $1.000 \mathrm{lux}, \mathrm{pH}$ entre $8,0-8,5$ e temperatura entre $25-28^{\circ} \mathrm{C}$.

Após 24 horas de incubação, um total de 1.200 naúplios instar II de $A$. franciscana foram contados e, em seguida, 100 indivíduos foram inoculados em cada um dos doze cones experimentais e tratados com diferentes combinações de S. maxima e S. cerevisiae. 
O experimento foi conduzido durante um período de 10 dias em um sistema experimental constituído de 12 cones de Imhoff. Assim como para a eclosão, os cones comportaram $1 \mathrm{~L}$ de água do mar filtrada em malha de $120 \mu \mathrm{m}$. As condições de salinidade, oxigênio dissolvido, pH e temperatura foram mantidas em níveis adequados. O fotoperíodo utilizado foi de 14 horas de escuridão por 10 horas de luminosidade (> 1.000 lux) (Sorgeloos et al., 1986).

Para assegurar a concentração de oxigênio dissolvido em níveis satisfatórios, e também, manter as partículas alimentares em suspensão, pontos de aeração de fundo foram instalados em cada cone. Uma renovação de 50 \% do volume de água foi realizada diariamente antes da primeira alimentação. Para isso, foi utilizado um sifão que apresentava, em uma de suas extremidades, uma malha de $120 \mu \mathrm{m}$ para deter o escape dos naúplios.

As dietas experimentais utilizadas consistiam de diferentes proporções da cianobactéria $S$. maxima, obtida a partir da descapsulação de comprimidos adquiridos em farmácias, e da levedura S. cerevisiae, obtida a partir de fermento seco adquirido em pontos de panificação. A composição nutricional da cianobactéria S. maxima e da levedura S. cerevisiae é apresentada na Tabela 1.

Tabela 1: Perfil nutricional da cianobactéria S. maxima e da levedura S. cerevisiae

\begin{tabular}{lcc}
\hline & S. maxima (\%) & S. cerevisiae (\%) \\
\hline Proteínas & $65,0-71,0$ & 39,0 \\
Lipídeos & 7,0 & 8,0 \\
Polissacarídeos & 4,6 & 34,1 \\
Cinzas & 9,0 & 3,1 \\
Outros & $8,4-14,4$ & 15,8 \\
\hline
\end{tabular}

Fonte: modificado de http://www.naturalways.com/spirul2.htm

Diariamente indivíduos de $A$. franciscana foram alimentados às $8 \mathrm{~h}(25 \mathrm{mg})$ e $17 \mathrm{~h}(25 \mathrm{mg})$ com diferentes combinações de S. maxima e $S$. cerevisiae, nas seguintes proporções: 100/0; 75/25; 50/50; 25/75 e 0/100. Antes das ofertas diárias, as dietas eram hidratadas por 5 min para desagregar as partículas. Todos os tratamentos foram realizados em duplicata. Adicionalmente, um controle negativo, também em duplicata, com ausência total de $S$. maxima e S. cerevisiae (0/0), foi utilizado. Desta forma, o experimento consistiu em seis tratamentos (100/0; 75/25; 50/50; $25 / 75,0 / 100$ e $0 / 0$ ).

Os dados abióticos apresentados nesse estudo foram coletados diariamente às $17 \mathrm{~h}$. As concentrações de oxigênio dissolvido e a temperatura da água foram obtidas por um oxímetro digital (modelo Y55). $\mathrm{O}$ pH foi determinado com o auxílio de um papel indicador; e a salinidade medida por um refratômetro com escala 0 \%o - 280 \%o (modelo S-28). Para verificar a luminosidade foi utilizado um luxímetro digital (modelo DX- 200).

Ao fim do experimento, $800 \mathrm{~mL}$ de cada cone teste foram sifonados para mais facilmente coletar os indivíduos de $A$. franciscana que permaneciam agrupados no volume de água restante. Uma vez coletados, estes foram estocados e conservados em frascos de $250 \mathrm{~mL}$ contendo $15 \mathrm{~mL}$ de formalina $5 \%, 175 \mathrm{~mL}$ de água do mar, além de $10 \mathrm{~mL}$ de clorofórmio. Essa última substância foi utilizada como anestésico, de modo a evitar a liberação de embriões ou cistos por parte das fêmeas.

Utilizando uma lupa estereoscópica, os critérios de avaliação sobrevivência, proporção juvenis/adultos, proporção sexual e performance reprodutiva (estado e modo reprodutivo; 
fecundidade) de $A$. franciscana foram determinados, em concordância com os valores percentuais e médios extraídos das duplicatas.

Para a sobrevivência, foi considerado o percentual dos indivíduos amostrados para cada tratamento dietético. Subsequentemente, os indivíduos foram agrupados em juvenis e adultos, e em machos e fêmeas. À semelhança da sobrevivência, a taxa percentual destes critérios foi determinada para cada tratamento.

Foram considerados juvenis os indivíduos desprovidos de órgãos genitais (hemipênis ou ovissaco) e de um acentuado dimorfismo sexual (antenas em forma de "pinça" nos machos); ou que quando presentes, estas estruturas eram pouco desenvolvidas. Por sua vez, os exemplares eram caracterizados como adultos a partir da emergência de um evidente dimorfismo sexual (antenas bastante desenvolvidas e em forma de "pinça" nos machos), assim como pelo completo desenvolvimento dos seus atributos genitais (hemipênis ou ovissaco).

Machos e fêmeas foram facilmente separados. Machos apresentavam um par de pênis (hemipênis) e o primeiro par de antenas era bastante desenvolvido com o seu característico formato de "pinça". As fêmeas eram facilmente identificadas pelo seu distintivo saco embrionário (ovissaco) e, geralmente, apresentavam-se com tamanho superior ao dos machos.

Já para o estado reprodutivo foi somente analisado o número de fêmeas, sejam elas ovígeras ou não-ovígeras. Foram consideradas fêmeas não-ovígeras as fêmeas imaturas que ainda não apresentam embriões ou cistos em seu ovissaco/oviduto. As fêmeas caracterizadas como ovígeras apresentavam alguma massa embrionária, sejam naúplios ou cistos, ainda em formação, ou já formados, em seu oviduto ou ovissaco.

Em seguida, as fêmeas ovígeras foram classificadas conforme seu modo reprodutivo em ovíparas (cistos no ovissaco), ovovivíparas (portando embriões) ou não-classificáveis (fêmeas com massa embrionária indistinguível em seu oviduto/ovissaco), e suas taxas subsequentemente determinadas. E finalmente, para se obter informações sobre a fecundidade média das fêmeas ovíparas ou ovovivíparas, o número de cistos ou embriões, respectivamente, foi determinado através da dissecação dos ovissacos.

\section{RESULTADOS}

Os resultados obtidos para o critério sobrevivência estão ilustrados na Figura 1, enquanto que os percentuais achados para os critérios maturidade sexual (juvenil/adulto) e proporção sexual estão sumarizados na Tabela 2.

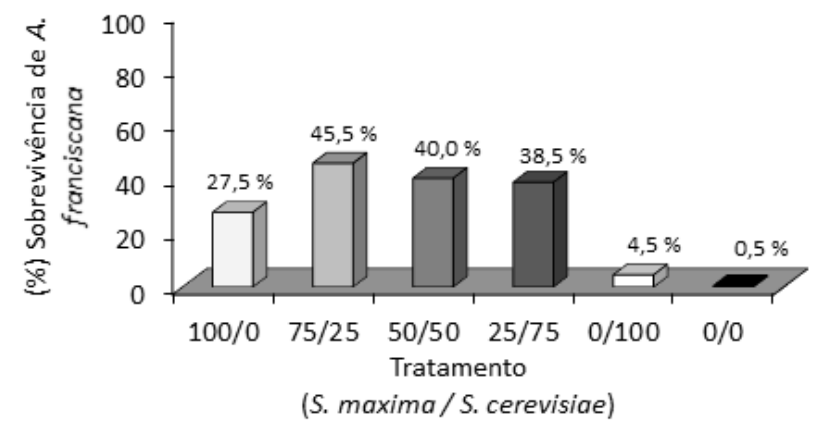

Figura 1: Sobrevivência média de $A$. franciscana obtida nos diferentes tratamentos dietéticos ao final do período experimental 
Conforme ilustra a Figura 1, os tratamentos 75/25 (45,5 \% $\pm 10,6), 50 / 50(40,0 \% \pm 25,5)$ e $25 / 75(38,5 \% \pm 47,4)$ apresentaram médias superiores àquelas obtidas nos tratamentos administrados integralmente à base de $S$. maxima $(27,5 \% \pm 2,1)$ e de $S$. cerevisiae $(4,5 \% \pm 0,7)$. Como esperado, o tratamento com ausência total das dietas experimentais apresentou sobrevivência média muito baixa $(0,5 \% \pm 0,7)$. Com exceção deste último tratamento, todos os demais apresentaram taxa percentual maior de indivíduos adultos sobre os juvenis, sendo as taxas $100 \%, 91,3 \%$ e $74 \%$ dos tratamentos $75 / 25$, 50/50 e 25/75, respectivamente, as mais expressivas (Tabela 2).

Quanto a proporção sexual, os resultados indicaram uma maior incidência de machos para todos os tratamentos, salvo o tratamento 0/100, no qual $50 \%$ dos indivíduos encontrados eram fêmeas, e o tratamento 0/0, no qual $100 \%$ dos indivíduos ficaram restritos à fase juvenil.

Ao fim do experimento, 93,2 \% $\pm 7,9$ das fêmeas apresentavam-se ovígeras no tratamento $75 / 25,72,4 \% \pm 18,4$ no tratamento $50 / 50,62,5 \% \pm 23,1$ no tratamento $100 / 0$ e $32,0 \% \pm 0,0$ no tratamento $25 / 75$, ao passo que o tratamento $0 / 100$ não apresentou fêmeas ovígeras, e o tratamento 0/0 não apresentou fêmeas (Tabela 3).

Tabela 2: Valores percentuais referentes a maturidade e proporção sexual de $A$. franciscana obtidos para os diferentes tratamentos dietéticos à base de $S$. maxima e $S$. cerevisiae.

\begin{tabular}{ccccc}
\hline $\begin{array}{c}\text { Tratamentos } \\
\text { Dietéticos } \\
\text { (S.maxima/S.cerevisae) }\end{array}$ & \multicolumn{2}{c}{$\begin{array}{c}\text { Maturidade Sexual } \\
\text { (\%) }\end{array}$} & \multicolumn{2}{c}{$\begin{array}{c}\text { Proporção Sexual } \\
\text { (\%) }\end{array}$} \\
\hline Juvenis & Adultos & Machos & Fêmeas \\
$100 / 0$ & 27,3 & 72,7 & 60 & 40 \\
$75 / 25$ & 0 & 100 & 51,6 & 48,4 \\
$50 / 50$ & 8,7 & 91,3 & 60,3 & 39,7 \\
$25 / 75$ & 26 & 74 & 56,1 & 43,9 \\
$0 / 100$ & 33,3 & 66,7 & 50 & 50 \\
$0 / 0$ & 100 & 0 & 0 & 0 \\
\hline
\end{tabular}

Com taxas superiores ou igual a $50 \%$, o modo reprodutivo ovovivíparo foi predominante, em relação ao ovíparo, em todos os tratamentos no qual esteve presente. Entretanto, o tratamento 100/0 apresentou $50 \%$ de fêmeas com o modo reprodutivo não-classificável.

Tabela 3: Valores percentuais referentes ao estado e modo reprodutivo das fêmeas de $A$. franciscana para os diferentes tratamentos dietéticos à base de S. maxima e S. cerevisiae.

\begin{tabular}{|c|c|c|c|c|c|}
\hline \multirow[t]{2}{*}{$\begin{array}{l}\text { Tratamentos } \\
\text { Dietéticos }\end{array}$} & \multicolumn{2}{|c|}{$\begin{array}{c}\text { Estado Reprodutivo } \\
(\%)\end{array}$} & \multicolumn{3}{|c|}{$\begin{array}{l}\text { Modo Reprodutivo das Fêmeas Ovígeras } \\
(\%)\end{array}$} \\
\hline & Não-ovígera & Ovígera & Ovíparo & Ovovivíparo & Não-classificável \\
\hline $100 / 0$ & 37,5 & 62,5 & 0 & 50 & 50 \\
\hline $75 / 25$ & 6,8 & 93,2 & 9,8 & 56,1 & 34,1 \\
\hline $50 / 50$ & 27,6 & 72,4 & 9,5 & 57,2 & 34,3 \\
\hline $25 / 75$ & 68 & 32 & 0 & 75 & 25 \\
\hline $0 / 100$ & 100 & 0 & 0 & 0 & 0 \\
\hline $0 / 0$ & 0 & 0 & 0 & 0 & 0 \\
\hline
\end{tabular}

A Tabela 4 apresenta uma visão geral dos valores médios e desvio padrão do número de fêmeas ovígeras e não-ovígeras (estado reprodutivo), assim como de ovíparas, ovovivíparas e nãoclassificáveis (modo reprodutivo), encontrado nos diferentes tratamentos dietéticos. Os valores 
médios do número de fêmeas, determinado para todos os tratamentos (duas repetições), em relação ao estado reprodutivo foi de 18,5 fêmeas não-ovígeras $(n=37)$ e 40 fêmeas ovígeras $(n=$ 80). Destas, 23 fêmeas ( $n=46)$ adotaram o modo ovovivíparo e apenas $3(n=6)$ adotaram o modo ovíparo, sendo a maior média, tanto de fêmeas ovíparas $(2,0 \pm 0,0)$ quanto ovovivíparas $(11,5 \pm$ $0,7)$, encontrada no tratamento $75 / 25$. Os resultados ainda apontaram que, em 14 fêmeas ovígeras ( $n=28)$, o modo reprodutivo não foi classificado.

Tabela 4: Valores médios do número de fêmeas de $A$. franciscana encontrados para o estado e modo reprodutivo nos diferentes tratamentos dietéticos à base de $S$. maxima e $S$. cerevisiae

\begin{tabular}{|c|c|c|c|c|c|}
\hline \multirow[t]{2}{*}{$\begin{array}{l}\text { Tratamentos } \\
\text { Dietéticos }\end{array}$} & \multicolumn{2}{|c|}{$\begin{array}{c}\text { Estado Reprodutivo } \\
\text { (média } \pm \text { DP) }\end{array}$} & \multicolumn{3}{|c|}{$\begin{array}{l}\text { Modo Reprodutivo das Fêmeas Ovígeras } \\
\text { (média } \pm \text { DP) }\end{array}$} \\
\hline & Não-ovígera & Ovígera & Ovíparo & Ovovivíparo & Não-classificável \\
\hline $100 / 0$ & $3,0 \pm 0,0$ & $5,0 \pm 4,2$ & $0,0 \pm 0,0$ & $2,5 \pm 2,1$ & $2,5 \pm 2,1$ \\
\hline $75 / 25$ & $1,5 \pm 2,1$ & $20,5 \pm 4,9$ & $2,0 \pm 0,0$ & $11,5 \pm 0,7$ & $7,0 \pm 4,2$ \\
\hline $50 / 50$ & $4,0 \pm 2,8$ & $10,5 \pm 12,0$ & $1,0 \pm 1,4$ & $6,0 \pm 7,1$ & $3,5 \pm 3,5$ \\
\hline $25 / 75$ & $8,5 \pm 12,0$ & $4,0 \pm 5,7$ & $0,0 \pm 0,0$ & $3,0 \pm 4,2$ & $1,0 \pm 1,4$ \\
\hline $0 / 100$ & $1,5 \pm 0,7$ & $0,0 \pm 0,0$ & $0,0 \pm 0,0$ & $0,0 \pm 0,0$ & $0,0 \pm 0,0$ \\
\hline 0/0 & $0,0 \pm 0,0$ & $0,0 \pm 0,0$ & $0,0 \pm 0,0$ & $0,0 \pm 0,0$ & $0,0 \pm 0,0$ \\
\hline $\begin{array}{l}\text { Total de } \\
\text { fêmeas }\end{array}$ & 18,5 & 40,0 & 3,0 & 23,0 & 14,0 \\
\hline
\end{tabular}

A fecundidade das fêmeas ovíparas variou de 63,0 cistos $\pm 2,8$ no tratamento $50 / 50$ a 105,0 cistos $\pm 33,2$ no tratamento $75 / 25$, ao passo que a fecundidade das fêmeas ovovivíparas variou de 45,3 embriões $\pm 8,9$ no tratamento $25 / 75$ a 92,0 embriões $\pm 34,9$ no tratamento $75 / 25$. Os tratamentos 0/100 e 0/0 não apresentaram fêmeas ovígeras, enquanto que o tratamento 100/0 com 75,6 embriões $\pm 32,9$ e 25/75 com 45,3 embriões \pm 8,9 só apresentaram fêmeas ovovivíparas (Figura 2).

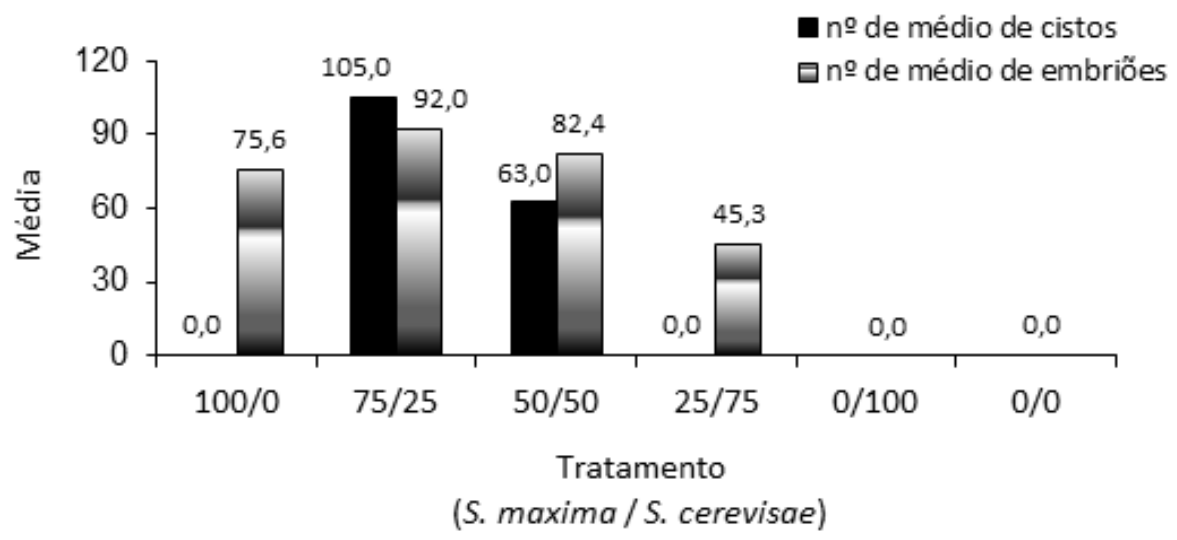

Figura 2: Fecundidade média de fêmeas ovíparas (cistos) e ovovivíparas (embriões) de $A$. franciscana nos diferentes tratamentos dietéticos.

Exceto a salinidade (mantida a $37 \%$ ), os dados abióticos obtidos no sistema experimental (temperatura, oxigênio dissolvido, luminosidade e $\mathrm{pH}$ ) foram mantidos em condições adequadas de cultivo (Sorgeloos et al., 1986) (Tabela 5). 
Tabela 5: Valores abióticos obtidos durante o período experimental.

\begin{tabular}{cccccc}
\hline Parâmetros & $\begin{array}{c}\text { Temperatura } \\
\left({ }^{\circ} \mathrm{C}\right)\end{array}$ & $\begin{array}{c}\text { Salinidade } \\
(\%)\end{array}$ & $\begin{array}{c}\text { OD } \\
(\mathrm{mg} / \mathrm{L})\end{array}$ & $\begin{array}{c}\text { Luminosidade } \\
(\mathrm{lux})\end{array}$ & $\mathrm{pH}$ \\
\hline Ideal & $25-28$ & 35 & $>5$ & $>1.000$ & $8,0-8,5$ \\
Obtido & 27,7 & 37 & - & 1.630 & 8,0 \\
\hline
\end{tabular}

\section{DISCUSSÃO E CONCLUSÃO}

De um modo geral, indivíduos de $A$. franciscana tratados com mistura entre as dietas obtiveram um melhor índice de sobrevivência. $O$ índice mais expressivo $(45,5 \% \pm 10,6)$ foi apresentado no tratamento em que $75 \%$ do conteúdo dietético era constituído de S. maxima. Conforme reduzia-se o conteúdo de S. maxima da dieta, os índices de sobrevivência foram decaindo, até os valores $4,5 \%( \pm 0,7)$ no tratamento $0 / 100$ e $0,5 \%( \pm 0,7)$ no tratamento $0 / 0$; os quais não apresentavam S. maxima em sua composição. Esse achado, somado ao fato de que a sobrevivência no tratamento integralmente à base de $S$. cerevisiae $(4,5 \% \pm 0,7)$ foi inferior àquele administrado exclusivamente com S. maxima $(27,5 \% \pm 2,1)$, leva a crer que esta possui um melhor valor alimentar (ingestibilidade, digestibilidade e/ou teor proteico) para $A$. franciscana que $S$. cerevisiae, muito embora a associação entre as duas dietas seja mais eficiente para manter o cultivo.

Os resultados do presente estudo assemelham-se aos de Coutteau et al. (1992), os quais testaram várias combinações dietéticas entre a microalga Dunaliella tertiolecta e $S$. cerevisiae no cultivo de $A$. franciscana e concluíram que a sobrevivência resultava em um melhor índice quando a dieta era constituída exclusivamente de $D$. tertiolecta $(89,0 \% \pm 6,0)$ ou quando havia associação de $25 \%$ desta microalga com $75 \%$ de $S$. cerevisiae $(88,0 \pm 7,0)$, que àquela constituída unicamente de $S$. cerevisiae. Os achados de Cavalcante et al. (2004) também corroboram os obtidos no presente trabalho. Em seu estudo, uma combinação de $25 \%$ de S. maxima com $75 \%$ da macroalga Gracilaria cervicornis micronizada resultou em um maior índice de sobrevivência de $A$. franciscana $(62,0 \% \pm 1,4)$ que àqueles com dietas integrais, constituídas exclusivamente de S. maxima $(25,5 \%$ $\pm 33,2)$ ou de $G$. cervicornis $(17,5 \pm 24,7)$. Todos esses resultados indicam que o cultivo pode ser viabilizado quando $A$. franciscana é tratada com dietas associadas.Sabendo que $S$. cerevisiae possui uma composição química constituída por elementos essenciais ao desenvolvimento de vários organismos, e que o valor alimentar de determinadas dietas, além do teor de proteínas e de outros constituintes, compreende a sua ingestibilidade e digestibilidade, pode-se assumir que provavelmente a ineficácia da dieta à base de $S$. cerevisiae tenha sido devido a uma baixa flutuabilidade e/ou estabilidade da levedura na coluna d'água, as quais implicariam em uma menor disponibilidade de células para $A$. franciscana. Esses fatos podem justificar o porquê de quantidades significativas da cianobactéria S. maxima (75\% e $50 \%$ ) serem requeridas para suprir o déficit de $S$. cerevisiae disponível no meio, e, por conseguinte, sustentar o cultivo. Coutteau et al. (1992) vão além, e admitem que uma certa quantidade de microalgas é também necessária para suplementar S. cerevisiae na dieta de A. franciscana e, desta forma, manter uma boa sobrevivência.

Resultados ainda melhores para a sobrevivência de $A$. franciscana foram alcançados por Coutteau et al. (1992), quando $S$. cerevisiae foi submetida a um processamento químico que consistia na remoção ou permeabilização de sua rígida parede celular, e uma adição de ácidos graxos insaturados (HUFA's). Certamente, o tratamento químico empregado favoreceu a uma 
maior flutuabilidade e estabilidade da levedura no meio de cultivo, logo maior ingestibilidade, além de um enriquecimento nutricional para os naúplios.

Contudo, o tamanho das partículas alimentares aparentemente foi satisfatório para $A$. franciscana, uma vez que essa apresentou uma boa sobrevivência, em todos os tratamentos, exceto naquele constituído unicamente por S. cerevisiae, o qual novamente poderia ser explicado por ocasião da estabilidade/flutuabilidade reduzida.

Considerando que para qualquer organismo atingir a maturidade sexual ele tem que assimilar, ao longo do seu desenvolvimento, partículas com um considerável valor alimentar, notase que a associação entre $S$. maxima e $S$. cerevisiae é, também, nutricionalmente mais viável para sustentar indivíduos de $A$. franciscana até os estádios adultos, uma vez que os resultados mais expressivos para a maturidade demonstraram uma maior predominância de adultos, em relação a juvenis, nos tratamentos que houve mistura (principalmente 75/25 e 50/50). Ainda que se tenha encontrado taxas superiores de adultos nos tratamentos com dietas integrais, os indivíduos submetidos a estas apresentaram um maior percentual para juvenis que àqueles tratamentos de associação entre as dietas.

Naquele mesmo estudo em 1992, Coutteau e colaboradores, afirmaram que $S$. cerevisiae suplementado com lipídios e vitaminas lipossolúveis presentes na microalga $D$. tertiolecta promove o crescimento de $A$. franciscana à estádios adultos como também diferenciações sexuais. Baseado nessa informação, pode-se admitir que a inteiração entre as dietas testadas no presente estudo, contém, além de um alto teor de proteínas, suficientes quantidades de lipídios, conforme mostra a Tabela 1, e possivelmente vitaminas requeridas para nutrir $A$. franciscana até estádios mais avançados.

A renovação diária de 50 \% no volume de água teve por finalidade minimizar o efeito do desenvolvimento de microorganismos junto às dietas, uma vez que o cultivo sem renovação da água pode propiciar condições satisfatórias para uma acelerada proliferação microbiana que pode servir como uma fonte alimentar alternativa para $A$. franciscana, e deste modo mascarar a deficiência da dieta.

James et al. 1987 apud Coutteau et al. (1992) cultivaram Artemia com a levedura marinha Candida, em baixa densidade e sem renovação de água, e alcançaram bons resultados. No entanto, as condições do cultivo foram satisfatórias para o desenvolvimento de "blooms" microbianos que serviu como um suplemento alimentar e, deste modo, omitiu a deficiência na dieta. Neste caso, o uso da levedura como uma fonte alimentar não permitiu uma autêntica avaliação do seu valor nutricional.

O efeito de microorganismos como fonte alimentar alternativa para naúplios de Artemia, foi evidenciado por Uchida et al. (1997). Inoculando Alteromonas espejiana, bactéria rica em proteínas, em um meio contendo talos formados a partir de Laminaria japonica (105-177 $\mu \mathrm{m}$ ), ele observou que $A$. espejiana degrada os talos em partículas ainda menores, se adere aos detritos formados e ainda suplementa tais detritos, que por sua vez são ingeridos pela Artemia.

As variáveis experimentais obtidas no sistema de cultivo foram mantidas em condições adequadas (Sorgeloos et al. 1986), e portanto, não interferiram nos critérios avaliados. Desta maneira, a predominância do modo reprodutivo ovovivíparo em relação ovíparo foi resultado, não só, de uma correta manutenção da qualidade da água, mas também de uma alimentação 
satisfatória, uma vez que a reprodução por oviparismo denota uma condição ambiental e/ou nutricional estressante.

Provavelmente, a condição pela qual algumas fêmeas inverteram seu modo reprodutivo para o oviparismo (tratamentos $75 / 25$ e 50/50) tenha sido uma deficiência na quantidade do alimento, uma vez que nestes tratamentos foi atingida uma alta sobrevivência. Além disso, opcionalmente, não foi ofertada uma quantidade maior das dietas à medida que os indivíduos cresciam. Outra possível condição que tenha feito as fêmeas adotarem tal estratégia pode ter sido uma predisposição genética, favorável ao desenvolvimento ovíparo, que poderia estar expressa em uma parte da população experimental; a qual só se confirmaria após estudos genéticos.

Nas mesmas condições de cultivo, Cavalcante et al. (2004), alimentaram A. franciscana com diferentes proporções de S. maxima e G. cervicornis, e igualmente encontraram uma maior predominância de ovoviviparismo em relação ao oviparaismo, sendo este último encontrado apenas nos tratamentos onde a sobrevivência foi elevada.

Os tratamentos nos quais $75 \%$ e $50 \%$ de S. maxima foi associada à S. cerevisiae, possivelmente, foram os mais bem sucedidos para estimular a cópula de $A$. franciscana, já que as fêmeas apresentaram valores expressivos tanto para o número médio de cistos $(92,0 \pm 34,9$ no tratamento $75 / 25$ e $82,4 \pm 20,2$ no tratamento $50 / 50)$ como para o de embriões $(105,0 \pm 33,2$ no tratamento $75 / 25$ e $63,0 \pm 2,8$ no tratamento $50 / 50$ ). Não obstante, o tratamento ofertado com $100 \%$ de S. maxima e o tratamento no qual $25 \%$ de S. maxima suplementou S. cerevisiae apresentaram uma alta fertilidade somente para as fêmeas ovovivíparas (dotadas de embriões). As fêmeas submetidas a dieta exclusivamente com $S$. cerevisiae não apresentavam cistos ou embriões, indicando que não foram fecundadas (fêmeas inférteis). Tais fatos levam a crer que dietas ofertadas integralmente à base de $S$. cerevisiae não são viáveis para assegurarem o comportamento pré-copulativo e a cópula de $A$. franciscana.

No entanto, os resultados obtidos para a fecundidade das fêmeas, sejam elas ovovivíparas ou ovíparas, neste último caso apenas nos tratamentos 75/25 e 50/50, apresentaram-se ótimos quando comparados com a descrição de Amat (1985), o qual relatou que a primeira postura de uma exemplar varia entre 10 e 30 embriões ou cistos, enquanto que as fêmeas de idade mais avançada, em perfeito estado reprodutivo, o número de embriões ou cistos, varia entre 100 e 400 , dependendo das condições do meio.

Contudo, os valores encontrados para o tratamento ofertado apenas com S. maxima e para os tratamentos com associações entre S. maxima e S. cerevisiae, demonstraram que tais tratamentos possuem dietas com um valor nutricional capaz de promover diferenciações sexuais, estimular a cópula e sustentar o cultivo de $A$. franciscana, uma vez que os indivíduos submetidos a estes tratamentos, não só apresentaram uma sobrevivência relativamente alta, mas também atingiram o estádio adulto e foram capazes de realizar a cópula.

Em conclusão, o estudo da utilização da cianobactéria S. maxima e da levedura S. cerevisiae como dietas complementares para $A$. franciscana, indicam que adequadas proporções entre as dietas testadas (75/25, 50/50 e 25/75), sobretudo com maior quantidade de $S$. maxima, atendem as perspectivas para o sustento de larvas e adultos de $A$. franciscana em sistemas de cultivos laboratoriais, e desta forma, são suficientemente viáveis para substituir, ou pelo menos suplementar, as microalgas vivas na nutrição desse microcrustáceo. 


\section{REFERÊNCIAS}

1. AMAT, F., 1985. Biología de Artemia. Instituto de acuicultura de Torre de la Sal. Inf. Tecn. Inst. Inv. Pesq./126-127. 59 p.

2. CAMARA, M. R., 1996. Artemia no Brasil: em busca de um modelo auto-sustentável de produção. Panorama de Aquicultura, 36: 16-19.

3. CAVALCANTE, P.H.O; SOUZA I.S; JUNIOR, M.A.F.C.; SOUZA, F.R.S.; CABRAL, T. M; CAMARA, M.R., 2004. Utilização da macroalga Gracilaria cervicornis e da cianobactéria Spirulina maxima como dietas complementares no cultivo de Artemia franciscana Kellogg (Crustacea; Anostraca) em escala laboratorial. In: VI Simpósio Brasileiro sobre Camarão - FENACAM, Natal, 2004. Resumos, Natal: ABCC, p. 31.

4. COUTTEAU, P., BRENDONCK, L., LAVENS, P., \& SORGELOOS, P., 1992. The use of manipulated baker's yeast as na algal substitute for the laboratory culture of Anostraca. Hydrobiologia, 234: 25-32.

5. PEDRAZA, G., X., 1989. Cultivo de Spirulina maxima para suplementación proteica. Livestock Research for Rural Development. Vol. I, N 1. Fundación CIPAV, Cali, Colômbia.

6. PERSOONE, G. \& SORGELOOS, P., 1980. General aspects of the ecology and biogeography of Artemia. In: G. Persoone, P. Sorgeloos, O. Roels, and Jaspers (Editores), The brine shrimp Artemia. Vol. 3. Universa Press, Wetteren, Bélgica, p. 3-24.

7. SORGELOOS, P., LAVENS, P., LEGER P., TACKAERT, W. \& VERSICHELE, D., 1986. Manual for the culture and use of brine shrimp Artemia in Aquaculture. 319 pp. University of Ghent. Belgica.

8. SZYPER, J. P., 2003. Live feeds: Production of the brine shrimp Artemia without natural sea water or microalgae. Aquaculture Extension Bulletin. P: 1-6.

9. TIZOL C., R., 1994. Uso de la levedura torula (Torulopsis utilis) en la obtención de biomassa de Artemia. An. Inst. Mar. Punta Betín, 23: 165-171. Santa Marta, Columbia.

10. UCHIDA, M., NAKATA, K., \& MAEDA, M., 1997. Introduction of detrital food webs into an aquaculture system by supplying single cell algal detritus produced from Laminaria japonica as a hatchery diet for Artemia nauplii. Aquaculture 154: 125-137.

11. VINATEA, L. A., 1998. Manual de producción de Artemias (quistes y biomasa) en módulos de cultivo. Proyeto II - A/2 "Localización, caracterización y evaluación del potencial extractivo de Artemia en Ibero-América con destino a la acuicultura". 59 p.

12. http://www.naturalways.com/spirul2.htm (Acessado em 06 de Fevereiro de 2012 às 13:25 horas). 